\title{
Neuronavigation-guided focused ultrasound (NaviFUS) for transcranial blood-brain barrier opening in recurrent glioblastoma patients: clinical trial protocol
}

\author{
Ko-Ting Chen ${ }^{1,2}$, Ya-Jui Lin ${ }^{1}$, Wen-Yen Chai ${ }^{3}$, Chia-Jung Lin ${ }^{4}$, Pin-Yuan Chen ${ }^{5}$, Chiung-Yin Huang ${ }^{6}$, \\ John S. Kuo ${ }^{7}$, Hao-Li Liu ${ }^{1,4}$, Kuo-Chen $\mathrm{Wei}^{6,8}$ \\ ${ }^{1}$ Department of Neurosurgery, Chang Gung Memorial Hospital at Linkou, Taipei; ${ }^{2} \mathrm{Ph} . \mathrm{D}$. Program in Biomedical Engineering, Chang Gung \\ University, Linkou, Taipei; ${ }^{3}$ Department of Diagnostic Radiology and Intervention, Chang Gung Memorial Hospital at LinKou, Linkou, Taipei; \\ ${ }^{4}$ Department of Electrical Engineering, Chang Gung University, Linkou, Taipei; ${ }^{5}$ Department of Neurosurgery, Chang Gung Memorial Hospital at \\ Keelung, New Taipei; ' ${ }^{6}$ epartment of Neurosurgery, New Taipei Municipal TuCheng Hospital, Chang Gung Memorial Hospital and Chang Gung \\ University, Linkou, Taipei; ${ }^{7}$ Department of Neurosurgery and Mulva Clinic for the Neurosciences, Dell Medical School, The University of Texas at \\ Austin, Austin, TX, USA; ${ }^{8}$ School of medicine, Chang Gung University, Linkou, Taipei \\ Contributions: (I) Conception and design: KC Wei, HL Liu, PY Chen, KT Chen, YJ Lin, JS Kuo; (II) Administrative support: CY Huang, KC Wei; (III) \\ Provision of study materials or patients: KC Wei, KT Chen, YJ Lin, HL Liu; (IV) Collection and assembly of data: KT Chen, YJ Lin, HL Liu; (V) Data \\ analysis and interpretation: KT Chen, HL Liu, WY Chai, CJ Lin; (VI) Manuscript writing: All authors; (VII) Final approval of manuscript: All authors. \\ Correspondence to: Kuo-Chen Wei. Department of Neurosurgery, Chang Gung Memorial Hospital at Linkou, No. 5, Fu Xing St., Guishan township, \\ Taipei. Email: kuochenwei@cgmh.org.tw.
}

\begin{abstract}
Background: Blood-brain barrier (BBB) limits over 95\% of drugs' penetration into brain, which has been a major obstacle in treating patients with glioblastoma. Transient BBB opening in glioblastoma (GBM) is feasible by combining focused ultrasound (FUS) with systemic infusion of microbubbles (MB). NaviFUS, a novel device that integrates neuronavigation and FUS-MB system, is able to intraoperatively direct the ultrasound energy precisely and repeatedly at targeted CNS areas. This clinical trial evaluates the safety and feasibility of NaviFUS in recurrent glioblastoma patients.

Methods: The study is a first-in-human, prospective, open-label, single-center, single-arm, dose escalation phase 1 clinical trial. A total of 6 patients will be enrolled. Patients will be enrolled into three groups, each group receiving an escalating dose of FUS energy (acoustic power is 4,8 , and $12 \mathrm{~W}$ ) with concomitant systemic microbubbles $(0.1 \mathrm{~mL} / \mathrm{kg})$ applied 1 week before surgical resection.

Results: Dynamic contrast-enhanced MRI will be obtained immediately and 24 hours after FUS procedures, while heavily $\mathrm{T} 2$-weighted sequence will be obtained to evaluate for any micro-hemorrhages. We anticipate that there will be minimal side effects associated with NaviFUS-mediated transient BBB opening.

Conclusions: Obtained results will support a planned phase 2 trial to evaluate whether NaviFUS can effectively enhance the delivery of chemotherapeutic agents and improve tumor control.
\end{abstract}

Keywords: Blood-brain barrier (BBB); brain tumor; focused ultrasound; glioblastoma; neuronavigation

Submitted Jan 06, 2020. Accepted for publication May 15, 2020.

doi: $10.21037 /$ atm-20-344

View this article at: http://dx.doi.org/10.21037/atm-20-344

\section{Introduction}

Glioblastoma (GBM) is the most frequently diagnosed and devastating adult primary brain tumor, with a high recurrence and 2-year mortality rate (1). GBMs are often poorly treated with many chemotherapies due to the bloodbrain barrier (BBB): which excludes more than $95 \%$ of large or small therapeutic agents and thereby prevents them from entering into malignant brain tumors $(2,3)$. The BBB is a 
complex structure that is sealed by tight junctions, which are unique to the brain microvasculature and protects the brain from exposure to potentially damaging substances $(4,5)$. BBB permeability can be increased by targeted focused ultrasound (FUS) combined with intravenous administered microbubbles $(6,7)$. The microbubbles reduce the ultrasonic energy needed to selectively disrupt the $\mathrm{BBB}$ and thus minimize damage to normal brain parenchyma $(8,9)$.

Targeted FUS exposure combined with microbubbles is capable of achieving local, temporal, and reproducible BBB opening with potential to enable and enhance drug delivery for brain diseases, such as delivering therapeutic agents locally for brain tumor treatment and improving clinical outcome $(6,7,10-14)$. Based on significant preclinical evidence, clinical trials of various devices that mediate FUSBBB opening have been initiated since 2014. A total of six trials have been registered involving glioblastoma patients using a variety of devices including SonoCloud ${ }^{\circledR}$ (CarThera Inc.), ExAblate ${ }^{\circledR}$ (InSightec Inc.) and NaviFUS ${ }^{\circledR}$ (NaviFUS Inc.), with and without chemotherapy agents (15). For now, three different types of therapeutic ultrasound devices, including the implanted ultrasound device $\left(\right.$ SonoCloud $\left.^{\circledR}\right)$, the extracorporeal fixed stereotactic frame-based magnetic resonance image (MRI)-guided device $\left(\right.$ Exablate $\left.^{\circledR}\right)$, and the frameless neuronavigation-guided device $\left(\mathrm{NaviFUS}^{\circledR}\right)$ are available on the market to treat and to explore their effectiveness in human clinical trials. Repeated BBB opening using implanted pulsed ultrasound device (SonoCloud ${ }^{\circledR}$ ) in combination with Sonovue ${ }^{\circledR}$ (dose: $\left.0.1 \mathrm{~mL} / \mathrm{kg}\right)$ at an acoustic pressure ranged from $0.5-1.1 \mathrm{MPa}$, has been shown to be safe and well tolerated in recurrent glioblastoma patients (16).

NaviFUS, a novel device integrating neuronavigation and FUS-MB system, is able to intraoperatively steer the transcranial burst-mode ultrasound energy precisely and repeatedly at targeted CNS areas (Figure 1). The purpose of this trial is to evaluate the safety and feasibility of using NaviFUS in treating recurrent glioblastoma patients. Based on previous reports demonstrating that focused ultrasound energy level of $4 \mathrm{~W}$ with clinically approved systemic dose of microbubbles $(0.1 \mathrm{~mL} / \mathrm{kg})$ can successfully open the BBB (17), the escalation focused ultrasound dose was started at this level. Moreover, the incremental ultrasound level was chosen to not exceed $0.8 \mathrm{MI}$ to minimize risk of erythrocyte extravasations in cerebral microvasculature (18). We present the following article in accordance with the SPIRIT reporting checklist (available at http://dx.doi.org/10.21037/ atm-20-344).

\section{Study goals and objectives}

The objectives of this study were to investigate the safety, feasibility and the tolerated dose of transient opening of the BBB by using the NaviFUS System in recurrent glioblastoma patients. We hypothesize that patients who receive ultrasonic energy delivered by NaviFUS System concomitant with systemic microbubbles administration will result in detectable transient $\mathrm{BBB}$ opening via dynamic contrast enhancing MRI (DCE-MRI).

\section{Study design}

This is a first-in-human, prospective, open-label, singlecenter, single-arm, dose escalation phase 1 clinical trial.

\section{Methods}

\section{Subjects}

\section{Inclusion criteria}

Adult patients over 20 years of age with recurrent glioblastoma (WHO grade IV) and are scheduled to undergo a surgical resection will be included. A recurrence is defined as a new contrast enhancing tumor, increase signal intensity change of non-enhancing lesion on T2/FLAIR (fluid-attenuated inversion recovery) $M R I$ and clinical deterioration on regular follow-up (19). The planned target region of interest (ROI) for FUS exposure are located close to the cortical surface, at least $20 \mathrm{~mm}$ under the skull. A peritumoral non-contrast enhanced region will be selected based on preoperative MRI in 3D model (including axial, sagittal and coronal views) by neurosurgeons, KT Chen or YJ Lin, and confirmed by a team who simulate and calculate the energy of FUS and planned dosage at targeted ROI. By doing so, a successful BBB disruption The ROI cannot be in the brainstem region, or critical functional motor or speech regions. Patient Karnofsky performance status (KPS) should be $>60$ (20). A patient consent will be obtained before their inclusions.

\section{Exclusion criteria}

Patients with a history of untreated AVM or cerebral aneurysm, or have acute hemorrhage or cyst within the region of interest (ROI) will be excluded. Patients with severe hypertension at screening (systolic blood pressure $>180 \mathrm{mmHg}$ or diastolic blood pressure $>100 \mathrm{mmHg}$ ), receiving anticoagulant or antiplatelet therapy within one week prior to study treatment will also be excluded. Detailed inclusion and exclusion criteria are listed in the Table 1. 


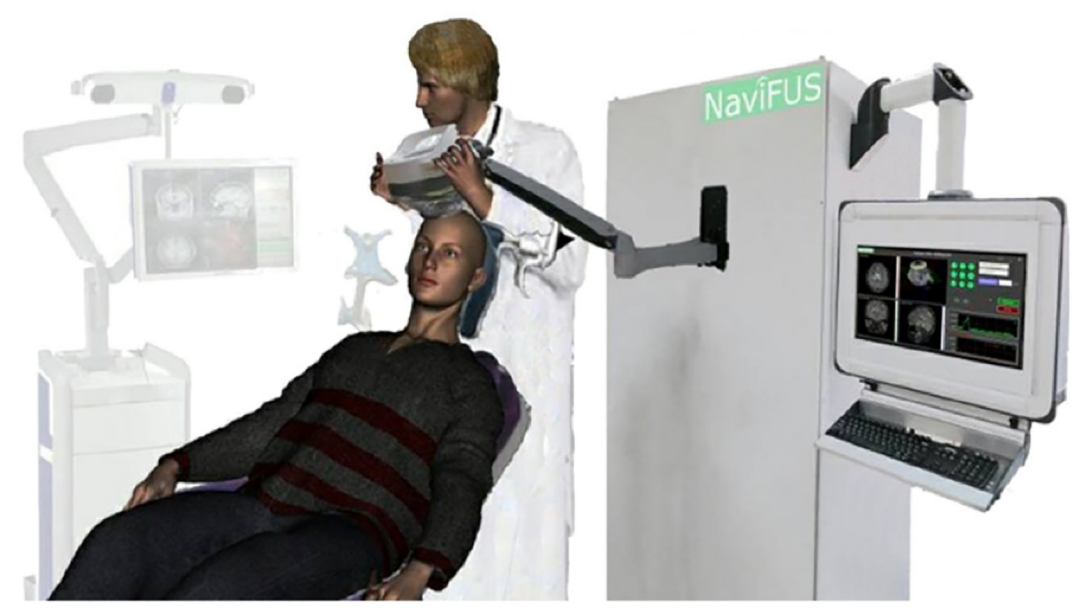

Figure 1 Schematic diagram of NaviFUS. The NaviFUS is a focused ultrasound phased array system in conjunction with clinically available neuronavigation to intraoperatively guide FUS energy deposit in the deep brain targeting position. A patient is fixed semi-rigidly without headpin system. A hand-held probe is connected to the power generator and working station. A doctor can steer the probe for precise transcranial targeted region under neuronavigation guidance. The whole procedure can be completed in one hour.

Table 1 Inclusion/exclusion criteria

Inclusion criteria
Adult male/female patients $>20$ years of age
Patients diagnosed with recurrent GBM and will undergo surgery
The ROI for FUS exposure in patients are located close to the cortex
surface with a minimum of 20 mm distance under the skull, and the ROI
is not in deep brain regions associated with critical brain functions, such
as in the brainstem, or motor or speech regions

If patients are already on radiotherapy, a gap of 7 days shall be Exclusion criteria

Patients diagnosed with arteriovenous malformation (AVM) or cerebral aneurysm

The receipt of an investigational drug, or participation in a drug research study within one month prior to the first FUS exposure

Known sensitivity/allergy to MRI contrast agents, CT contrast agents, SonoVue ${ }^{\circledR}$, or any of its components

maintained between the last day of radiotherapy and the day of screening

If patients are already receiving steroids treatment, then the steroid dose should be on a stable and unchanged for at least 7 days prior to screening

Life expectancy $\geq 3$ months

Adequate hepatic, renal, coagulation, and hematopoietic function as follows: (I) Hemoglobin $\geq 10 \mathrm{~g} / \mathrm{dL}$; (II) Platelets $\geq 100,000 / \mathrm{mm}^{3}$; (III) Neutrophils $\geq 1,500 / \mathrm{mm}^{3}$; (IV) Normal creatinine clearance $\geq 50 \mathrm{~mL} / \mathrm{min}$; (V) Alanine transaminase (ALT) $<3 \times$ upper limit of normal (ULN); (VI) Aspartate transaminase (AST) <3× ULN; (VII) Prothrombin time $\leq 1.2 \times$ ULN; (VIII) International Normalized Ratio (INR) $<1.5$; (IX) Bilirubin $<2 \times$ ULN
Patients who have acute hemorrhage or cyst within the ROI

Severe hypertension at screening (Systolic blood pressure $>180 \mathrm{mmHg}$ or diastolic blood pressure $>100 \mathrm{mmHg}$ on medications)

Receipt of anticoagulant (e.g., warfarin) or antiplatelet (e.g., aspirin) therapy within one week prior to study treatment, or other drugs known to increase risk or hemorrhage (e.g., Avastin) within one month prior to study treatment

GBM, glioblastoma multiforme; FUS, focused ultrasound; ROI, region of interest; MRI, magnetic resonance image; CT, computed tomography; ULN, upper limit of normal. 


\section{Study description}

All eligible patients will be randomly assigned to 3 groups $(\mathrm{n}=6 ; 2$ patients per group) and will receive defined ultrasound exposure doses (in MI, mechanical index) generated from the NaviFUS System to transiently open the $\mathrm{BBB}$ in recurrent GBM patients who are planning to undergo surgery within 2 weeks. The focused ultrasound (FUS) dose is selected based on the results of pre-clinical Good Laboratory Practice (GLP) safety studies and other non-GLP primate studies (21).

For the selection of energy dosage, the initial FUS dose is selected to be no exceeding $0.48 \mathrm{MI}$ at target, because this corresponds to the threshold for $\mathrm{BBB}$ opening in previous nonclinical studies $(17,22)$. The highest dose of $0.68 \mathrm{MI}$ is supported by the GLP safety tests in rats and beagle dogs. Two other non-GLP preclinical toxicology study references also supported that no exceeding $0.68 \mathrm{MI}$ at target was a marginal dose for safety $(21,23)$. Furthermore, the 2016 clinical study using Sonocloud reported the application of five different ultrasound doses on human brains. There was no ultrasound related DLT was found in 0.5 to $1.1 \mathrm{MI}$ (16). Finally, energy doses of $0.48,0.58$ and $0.68 \mathrm{MI}$ were applied.

The trial protocol will be as follows: The first group of patients ( $\mathrm{n}=2$, FUS dose: $0.48 \mathrm{MI}$ ) will receive the lowest FUS dose, with each patient subject receiving one cycle of up to $4 \mathrm{~W}$ NaviFUS treatment. NaviFUS treatment will be conducted with the following exposure conditions: total exposure time: $120 \mathrm{~s}$, focal scanned matrix: $3 \times 3$ (gap between adjacent focus: $5 \mathrm{~mm}$ ), pulse repetition frequency: $9 \mathrm{~Hz}$, burst length/focus exposure: $10 \mathrm{~ms}$. After FUS treatment (day 0), patients will have initial follow-up at post-NaviFUS $7 \pm 3$ days (depends on the planned date of resection surgery) follow-up visit to evaluate post-NaviFUS treatment toxicities. After surgery, patients will be followed for 28 days, including $2^{\text {nd }}$ and $3^{\text {rd }}$ follow-up visits at postoperative days $14 \pm 6$ and $28 \pm 6$, respectively.

The data and safety monitoring board (DSMB) will review the study data when the $1^{\text {st }}$ group of trial subjects complete a post-NaviFUS treatment day $7 \pm 3$ followup visit, and provide recommendations regarding study continuation, termination, or other modifications based on any observed adverse effects of NaviFUS intervention. Dose escalation will occur only after DSMB review and approval that the $1^{\text {st }}$ FUS dose group trial has less than one FUS-related dose-limiting toxicity (DLT) (during post-FUS treatment days $7 \pm 3$ ). The $2^{\text {nd }}$ group will then be enrolled (number of subjects $=2$, with each subject receiving one cycle of up to $8 \mathrm{~W}$ NaviFUS treatment, FUS dose: 0.58 MI). After another DSMB review and approval, then the $3^{\text {rd }}$ group will be enrolled (subject number $=2$, with each subject receiving one cycle of up to $12 \mathrm{~W}$ NaviFUS treatment, FUS dose: $0.68 \mathrm{MI})$. After completion of all three test groups, a final report will be prepared and submitted for presentation and publication.

\section{Outcome measures and follow-up}

\section{Primary endpoints}

Evaluate safety of transient BBB opening by the NaviFUS System in recurrent glioblastoma patients: DLT, adverse effects (AEs), physical examination, neurological examination, KPS, mini-mental state examination (MMSE), vital signs, and clinical laboratory tests.

\section{Secondary endpoints}

Evaluate tolerated ultrasound power level with the NaviFUS system for transient BBB disruption. If patients cannot tolerate or show DLTs for a particular FUS level, then the previous lower FUS dose group will be considered as the maximum tolerated dose.

\section{Discussion}

\section{Trial status}

Patient recruitment is scheduled to be completed by September 2019. Data analysis will be performed subsequently and the final reports will be disseminated after the last follow up for the $6^{\text {th }}$ patient subject.

\section{Safety considerations}

All aspect of the study shall be conducted under Good Clinical Practice (GCP) guidelines stipulated by the regulatory agency, Taiwan Food and Drug Administration, and will be monitored by qualified individuals designated by the sponsor. All trial monitoring will be conducted in accordance with GCP and standard compliance operating procedures with applicable government regulations. The investigators agree to assist and facilitate access of monitors to the clinical supplies and storage area, and to the clinical files of all study patients.

\section{Follow-up}

All patients will be followed until 37 days after NaviFUS 


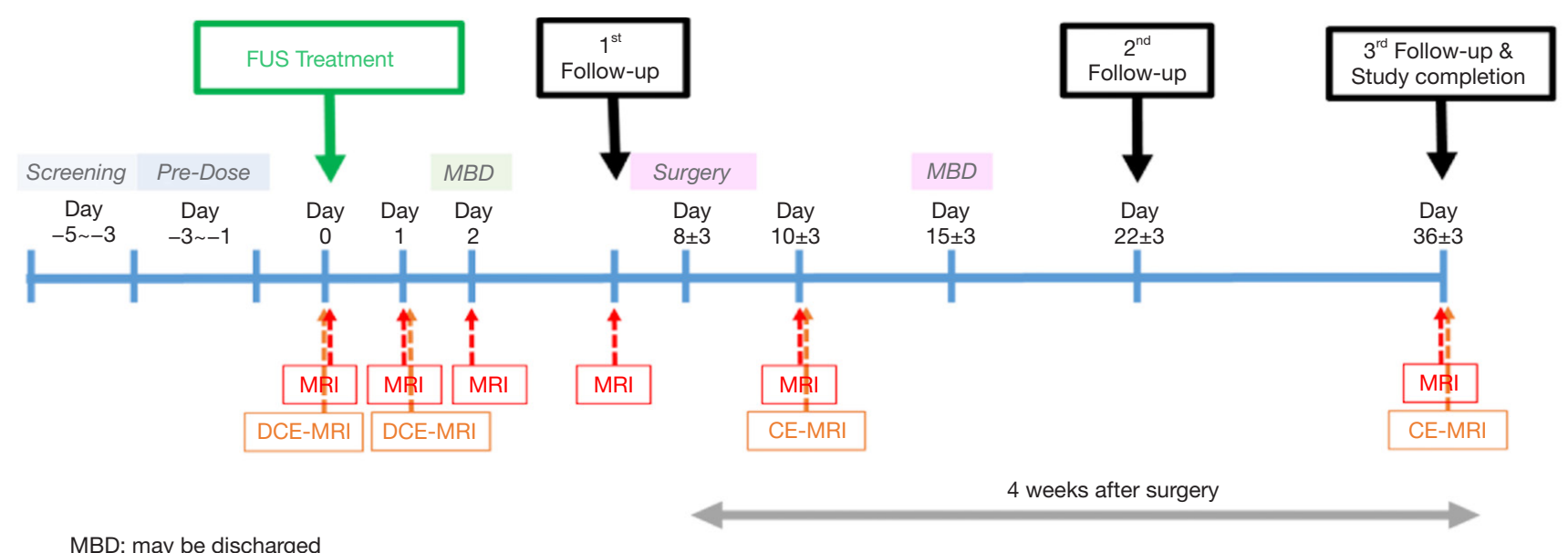

Figure 2 Study protocol for the clinical trial.

treatment or 30 days after definitive resection surgery. DCE-MRI to evaluate the effectiveness of BBB opening will be performed immediately after NaviFUS treatment and 24 hours post-FUS. Standard pre- and post-surgical brain gadolinium contrast enhanced-MRI will be scheduled (Figure 2). Clinical visits with history, physical and neurological examinations will be routinely performed to assess functional status and clinical well-being. Detailed instructions are given to each patient and their family in the event of an emergency. All patients and their caretakers are educated about reporting adverse events that may be related to the treatment protocol.

\section{Data management and statistical analysis}

Descriptive statistics will be provided for selected demographic, safety data for each FUS dose and visit. Descriptive statistics on continuous measurements will include means, medians, standard deviations, and ranges, while categorical data will be summarized using frequency counts and percentages.

All subjects who receive a FUS dose will be included in the safety analyses. Summary statistics will be conducted to describe the safety variables, including adverse events (AEs), as reported throughout the course of the trial, per treatment group. Pre-dose and post-dose findings of physical examination, neurological examination, KPS, MMSE, vital sign variables, laboratory data, and MRI will be listed individually and summarized; values outside the normal range will be listed. The AEs will be summarized as counts, frequencies, and grade at each dose level.

In addition, a list of subjects who withdraw early from this study will be provided. It will include the reason and timing of a subject's withdrawal. Similarly, the reason any subject is excluded from an analysis set will also be provided. In addition, significant known protocol deviations will be noted for individual subjects.

A data monitoring committee composed of members independent from the sponsor and competing interests will monitor and interim reports after each escalating dosage and it would their decision to determine whether this trial should or should not continue the trial and dose-escalating strategy.

\section{Quality assurance}

To ensure accurate, complete, and reliable data, NaviFUS Corporation or its representatives will provide standard operating procedures (SOPs) and instructional materials to the study site, as appropriate. To ensure the safety of study participants and to ensure accurate, complete, and reliable data, the investigator shall enter and keep data in the Case Report Forms: including laboratory data, patient histories, treatment regimens, physical examination, concomitant medications/therapy, and any AEs in the patient files as original source documents for the study.

\section{Expected outcomes of the study}

Based on preclinical studies, we expect that the chosen doses of NaviFUS treatment will not be associated with major AEs, such as hemorrhage related to BBB opening, or central nervous system effects related to ultrasound stimulation. We anticipated that NaviFUS will successfully 
and transiently open BBB as demonstrated by DCE-MRIs immediately post-treatment and 24 hours post-treatment. We anticipate establishing a safe maximum tolerated FUS dose will be determined via testing for effectiveness of BBB opening and any AEs noted by participated subjects.

\section{Duration of the project}

The study plans to enroll subjects within 8 to 12 months for the first phase of this clinical trial. If safety and tolerated dose are established, a phase II clinical trial will be planned and initiated.

\section{Project management}

The study is being led by the principal investigator. He will oversee and supervise the entire study team in performing all aspects of study for this clinical trial. Data interpretation and dissemination of results will be managed under his direct supervision.

\section{Acknowledgments}

Funding: The clinical trial and research leading to these results were sponsored by Chang-Gung Memorial Hospital at Linkou, Taiwan (CMRPG3J0091), No. 5, Fuxing St., Guishan Dist., Taoyuan City 333, Taiwan; Ministry of Science and Technology, Taiwan (MOST 107-2119-M-182001), 106, Sec. 2, Heping E. Rd., Taipei 10622, Taiwan; National Health Research Institute (NHRI-EX10810502NI) and 35 Keyan Road, Zhunan, Miaoli County 35053, Taiwan; NaviFUS corporation 12F., No. 246, Sec. 3, Chengde Rd., Datong Dist., Taipei City 103, Taiwan.

\section{Footnote}

Reporting Checklist: The authors have completed the SPIRIT reporting checklist. Available at http://dx.doi.org/10.21037/ atm-20-344

Conflicts of Interest: All authors have completed the ICMJE uniform disclosure form (available at http://dx.doi. org/10.21037/atm-20-344). Dr. Liu reports non-financial support from NaviFUS, during the conduct of the study. The device was developed by NaviFUS Inc. The clinical trial and research leading to these results were sponsored by Chang-Gung Memorial Hospital at Linkou, Taiwan (CMRPG3J0091), Ministry of Science and Technology,
Taiwan (MOST 107-2119-M-182-001), National Health Research Institute (NHRI-EX108-10502NI) and NaviFUS.

Ethical Statement: The authors are accountable for all aspects of the work in ensuring that questions related to the accuracy or integrity of any part of the work are appropriately investigated and resolved. This study shall be conducted in accordance with the Declaration of Helsinki, GCP guidelines, and all applicable laws and regulations of the Taiwan FDA. The study was approved by institutional Review Board of Chang-Gung Medical Foundation (No. 201800111A0) and informed consent was taken from all the patients. Written informed consent was obtained from the patient for publication of this study and any accompanying images. A copy of the written consent available for review by the Editor-in-Chief of this journal. All personal information about enrolled participants will be maintained and will not be shared or only in a de-identified form in order to protect confidentiality during and after the trial.

Open Access Statement: This is an Open Access article distributed in accordance with the Creative Commons Attribution-NonCommercial-NoDerivs 4.0 International License (CC BY-NC-ND 4.0), which permits the noncommercial replication and distribution of the article with the strict proviso that no changes or edits are made and the original work is properly cited (including links to both the formal publication through the relevant DOI and the license). See: https://creativecommons.org/licenses/by-nc-nd/4.0/.

\section{References}

1. Behin A, Hoang-Xuan K, Carpentier AF, et al. Primary brain tumours in adults. Lancet 2003;361:323-31.

2. Pardridge WM. The blood-brain barrier: bottleneck in brain drug development. NeuroRx 2005;2:3-14.

3. Pardridge WM. Drug and gene delivery to the brain: the vascular route. Neuron 2002;36:555-8.

4. Brightman MW, Reese TS. Junctions between intimately apposed cell membranes in the vertebrate brain. J Cell Biol 1969;40:648-77.

5. Hawkins BT, Davis TP. The blood-brain barrier/ neurovascular unit in health and disease. Pharmacol Rev 2005;57:173-85.

6. Hynynen K, McDannold N, Sheikov NA, et al. Local and reversible blood-brain barrier disruption by noninvasive focused ultrasound at frequencies suitable for trans-skull sonications. Neuroimage 2005;24:12-20. 
7. Hynynen K, McDannold N, Vykhodtseva N, et al. Noninvasive MR imaging-guided focal opening of the blood-brain barrier in rabbits. Radiology 2001;220:640-6.

8. Hynynen K. MRI-guided focused ultrasound treatments. Ultrasonics 2010;50:221-9.

9. McDannold N, Zhang Y, Vykhodtseva N. Blood-brain barrier disruption and vascular damage induced by ultrasound bursts combined with microbubbles can be influenced by choice of anesthesia protocol. Ultrasound Med Biol 2011;37:1259-70.

10. Hynynen K, McDannold N, Vykhodtseva N, et al. Noninvasive opening of BBB by focused ultrasound. Acta Neurochir Suppl 2003;86:555-8.

11. McDannold N, Vykhodtseva N, Raymond S, et al. MRIguided targeted blood-brain barrier disruption with focused ultrasound: histological findings in rabbits. Ultrasound Med Biol 2005;31:1527-37.

12. Mesiwala AH, Farrell L, Wenzel HJ, et al. High-intensity focused ultrasound selectively disrupts the blood-brain barrier in vivo. Ultrasound Med Biol 2002;28:389-400.

13. Wei KC, Chu PC, Wang HY, et al. Focused ultrasoundinduced blood-brain barrier opening to enhance temozolomide delivery for glioblastoma treatment: a preclinical study. PLoS One 2013;8:e58995.

14. Liu HL, Hsu PH, Lin CY, et al. Focused Ultrasound Enhances Central Nervous System Delivery of Bevacizumab for Malignant Glioma Treatment. Radiology 2016;281:99-108.

15. Chen KT, Wei KC, Liu HL. Theranostic Strategy of Focused Ultrasound Induced Blood-Brain Barrier Opening for CNS Disease Treatment. Front Pharmacol 2019;10:86.

Cite this article as: Chen KT, Lin YJ, Chai WY, Lin CJ, Chen PY, Huang CY, Kuo JS, Liu HL, Wei KC. Neuronavigation-guided focused ultrasound (NaviFUS) for transcranial blood-brain barrier opening in recurrent glioblastoma patients: clinical trial protocol. Ann Transl Med 2020;8(11):673. doi: 10.21037/atm20-344
16. Carpentier A, Canney M, Vignot A, et al. Clinical trial of blood-brain barrier disruption by pulsed ultrasound. Sci Transl Med 2016;8:343re2.

17. McDannold N, Vykhodtseva N, Hynynen K. Bloodbrain barrier disruption induced by focused ultrasound and circulating preformed microbubbles appears to be characterized by the mechanical index. Ultrasound Med Biol 2008;34:834-40.

18. Chai WY, Chu PC, Tsai MY, et al. Magnetic-resonance imaging for kinetic analysis of permeability changes during focused ultrasound-induced blood-brain barrier opening and brain drug delivery. J Control Release 2014;192:1-9.

19. Wen PY, Macdonald DR, Reardon DA, et al. Updated response assessment criteria for high-grade gliomas: response assessment in neuro-oncology working group. J Clin Oncol 2010;28:1963-72.

20. Mor V, Laliberte L, Morris JN, et al. The Karnofsky Performance Status Scale. An examination of its reliability and validity in a research setting. Cancer 1984;53:2002-7.

21. McDannold N, Arvanitis CD, Vykhodtseva N, et al. Temporary disruption of the blood-brain barrier by use of ultrasound and microbubbles: safety and efficacy evaluation in rhesus macaques. Cancer Res 2012;72:3652-63.

22. Hynynen K, McDannold N, Vykhodtseva N, et al. Focal disruption of the blood-brain barrier due to $260-\mathrm{kHz}$ ultrasound bursts: a method for molecular imaging and targeted drug delivery. J Neurosurg 2006;105:445-54.

23. Horodyckid C, Canney M, Vignot A, et al. Safe long-term repeated disruption of the blood-brain barrier using an implantable ultrasound device: a multiparametric study in a primate model. J Neurosurg 2017;126:1351-61. 